\title{
Polare Erregung und Hemmung an Arterien.
}

\author{
Von \\ Paul v. Liebermann.
}

(Aus dem tierphysiologischen Laboratorium der Universität Kopenhagen.)

(Eingegangen am 28. Juli 1921.)

Gegenwärtige Mitteilung hat den Zweck, einen Effekt bekannt zu machen, der besonders geeignet erscheint, die GesetzmäBigkeiten der polaren Stromeswirkungen in ihrer Erscheinung am glattmuskeligen Organ auf bequeme Art zu demonstrieren und nach manchen Seiten hin zu untersuchen. Da, wie ich nach einem vorläufigen Abschluß der Arbeit finde, das Verhalten der Erregungserscheinungen an der Kathode bis ins einzelne mit den grundlegenden Beobachtungen Engelmanns am Ureter übereinstimmt, und da ausführliche Arbeiten über den ganzen Gegenstand, namentlich von Bieder mann und seinen Mitarbeitern, auch aus neuerer Zeit vorliegen, so würde ich von einer Veröffentlichung abgesehen haben, wenn ich nicht glaubte, daß das von mir gewählte Untersuchungsobjekt der Beobachtung einige besondere Vorteile gewährt und eine Mitteilung daher als Beitrag zur Methodik dieses Gebietes erwünscht sein möchte.

Die mikroskopische Beobachtung an den Arterien der Schwimmhaut des Frosches (R. fusca), deren ich mich bedient habe, empfiehlt sich vor allem dadurch, daß man am unverletzten Tier arbeitend von den Ver. änderungen der Frregbarkeit, wie sie am bloßgelegten oder freipräparierten Organ eintreten können, unbehelligt bleibt und die Versuche dahєr beliebig oft im natürlichen Zustande des Gewebes wiederholen kann. Besonders günstig aber ist der Umstand, daß die blutdurchströmte Arterie unter dauerndem Druck von innen steht, so daß sich jede Erschlaffung der Wand als Dilatation kundgeben muß und die hemmenden Wirkungen des elektrischen Stromes auf diese Weise ebenso sinnfällig werden wie die erregenden, ohne daß man es nötig hätte, die Muskelfasern vorher künstlich in den Zustand der Dauerkontraktion zu versetzen. Dieser Vorteil kommt uns freilich auch bei entsprechenden Beobachtungen am Herzen zugute, an dessen Kammermuskel durch die Anode eine lokale Diastole hervorgebracht wird; doch ist hier das Bild der Wirkung durch die Schwankungen des Kammerinnendruckes kompliziert').

Man überzeugt sich zunächst, daß an der Kathode Zusammenziehung an der Anode Erweiterung der Arterien durch den Strom hervorgerufer wird. Diese Effekte sind in großer Stärke zu erhalten; es gelingt leicht

1) Über diesen besonders durch Biedermanns Arbeiten bekannt gewordenes Effekt vgl. neuerdings Palladin, Über die anodische Wirkung des konstantes Stromes auf das Froschherz, Zeitschr. f. Biologie 62, 418. 191.3. 
die Arterie durch Einwirkung der Kathode zum Verschluß zu bringen, durch Einwirkung der Anode aufs Mehrfache ihres Durchmessers zu erweitern. Doch läßt sich die Wirkung durch Veränderung der Stromstärke bis zu kaum merklicher Reaktion abstufen. An den Venen habe ich keine Wirkung beobachten können.

Die Latenz weist für beide Wirkungen eine bedeutende Abhängigkeit von der Stromstärke auf, indem sie mit zunehmender Stromstärke kürzer wird. Bei gleichem Strom fand ich sie oft für die anodische Wirkung länger als für die kathodische, doch muß ich es unentschieden lassen, ob dieser Unterschied reelle Bedeutung hat, da vermutlich der Beginn einer Verengerung schärfer als der einer Erweiterung zu erkennen ist. Die kürzesten Werte, die ich beobachtete, betrugen etwa 1 Sek.für die kathodische, 2 Sek. für die anodische Wirkung (nicht in demselben Versuch); doch haben die Zahlen nach dem Gesagten keine absolute Bedeutung.

Sowohl die kathodische als die anodische Reaktion ist bei nicht zu geringen Stromstärken Dauerwirkung des Stromes, sie bleibt bestehen, solange der Strom geschlossen ist; ich habe diesen Versuch bis zu einer Viertelstunde ausgedehnt. Für die kathodische Wirkung ist allerdings zu bemerken, daß der constrictorische Krampf bei anhaltender Durehströmung in der Regel um ein weniges nachläßt. Mit ihrem Charakter als Dauerwirkungen steht es im Einklang, daß es mir nicht gelang, die Erscheinungen durch Einschleichen des Stromes hintanzuhalten. Ich hatte einen gewöhnlichen Schieberwiderstand im Kreis und verwendete einen Zweigstrom zur Reizung, so daß die wirksame Spannung durch Sehleifen des Schiebers zu verändern war; diese Anordnung genügte, den Strom in einen Nerven ohne Wirkung einzuführen, während die Arterien reagierten. Doch ist es freilich möglich, ja nach den Angaben Engelmanns wahrscheinlich, daß bei noch allmählicherem Anwachsen der Intensität die Wirkung, wenigstens die kathodische, ausbleiben würde ${ }^{1}$ ).

Der Zeitverlauf der Reaktion ist, so wie dies von der Latenz gilt, je nach der Stromstärke verschieden, zumindest gilt dies für die constrictorische Wirkung der Kathode, die bei den höheren Stromintensitäten ibre volle Stärke sehr rasch erreicht, bei schwächeren Strömen dagegen einen gedehnten Verlauf nimmt und ganz allmählich ansteigt. Schließt man den Strom nur für kurze Zeit - in der Regel bielt ich ihn bis zur vollen Entwicklung der Reaktion geschlossen -, so kann man kathodische Verengerung, aber auch anodische Erweiterung bei schon geöffnetem Stromkreis noch zunehmen sehen, ehe sie von den gleich zu beschreibenden gegensinnigen Nachwirkungen abgelöst werden.

War nämlich die Arterie kathodischer Einwirkung ausgesetzt gewesen, so wird sie nach Unterbrechung, ja selbst nach ,,Ausschleichen “

1) Beiträge zur allgemeinen Muskel- und Nervenphysiologie. Dieses Arch. 3, 247. 1870. Vgl. Punkt D, S. 268. 
des Stromes in der Regel erweitert, ebenso kräftig als ob die Anode einwirkte. Diese Nachwirkung ist anhaltend, und geht nur ganz allmählich zurück. Entsprechend sieht man nach anodischer Einwirkung oft starke Constriction bis zum völligen Verschluß, der sehr lange anhalten kann. Hier muß jedoch erwähnt werden, daß diese Nacheffekte nicht immer in die Augen springen, ja vielleicht auch nicht immer zugegen sind. Nach kathodischer Einwirkung kommt es vor, daß die Arterie noch eine Zeitlang verengt bleibt; besonders aber ist eine solche gleichsinnige Nachdauer nach anodischer Einwirkung das gewöhnliche, indem die Arterie nach der Öffnung des Stromes minutenlang erweitert bleibt und sich nur sebr allmählich wieder verengt.

Es darf wohl an dieser Stelle darauf hingewiesen werden, daß sich dies nach dilatatorischer Nerveneinwirkung ebenso verhält. Schon aus den Beobachtungen von Goltz mit Freusberg geht es hervor ${ }^{1}$ ); von seinen Versuchen sind die über Nervendurchschneidung hier nicht zu verwerten wegen der andauernden Reizwirkung des Schnittes, wohl aber der Versuch mit elektrischer Reizung (S. 184), da hier die Pfote der gereizten Seite nach 6 Stunden noch etwas wärmer als die andere war. B a yliss teilt in seiner Arbeit, die den Nachweis der antidromen Effekte enthält, das gleiche mit ${ }^{2}$ ), und wenn er auch geneigt ist, die aus seinen Kurven ersichtliche besonders lange Nachdauer als einen von der Methode vorgetäuschten Scheineffekt anzusehen, so wird doch ein Teil davon reeller Natur gewesen sein; er weist hierauf selbst als eine gewöhnliche Erfahrung bei gefäßerweiternden Nerveneinflüssen hin, und sie hat auch am Frosch für die entsprechende Wirkung der Hinterwurzelfasern Geltung. Läßt nun auch das Angeführte für sich allein keine weitgehenden Schlüsse über die Natur der gefäßerweiternden Nervenwirkung $z u$, so haben wir doch die Übereinstimmung der anodischen und der nervösen Hemmungswirkung in dieser Einzelheit nicht unerwähnt lassen wollen. Ich möchte hinzufügen, daß die Wirkung der Kathode aufgehoben wird., wenn die Arterien extrem erweitert werden durch Reize, die ohne Zweifel an den sensibeln, also dilatatorischen Nervenelementen angreifen. So kann man durch wasserentziehende Mittel, wie konzentrierte Kochsalzlösung (etwa 8 proz.), oder Glycerin, wenn ein Tropfen davon auf die Schwimmhaut gebracht wird, die Arterien zu äußerster Erweiterung veranlassen, und während dieses Zustandes ist die Kathode unwirksam; die Anode bewirkt natürlich anch keine weitere Veränderung. Ähnliches erhält man durch mechanischen Reiz (Kneifen), wodurch ebenfalls die Arterien erweitert werden;

1) Über gefäßerweiternde Nerven. Pflügers Arch.f. d. ges. Physiol., 9, 174. 1874.

$\left.{ }^{2}\right)$ On the origin from the spinal cord of the vaso-dilator fibres of the hind. limb, and on the nature of these fibres. Journ. of Physiol. 26, 173. 1900-1901. Vgl. S. 180-181. 
auch dadurch wird die Kathodenwirkung zumindest stark beeinträchtigt. $\mathrm{Ob}$ hieran freilich der besondere Hemmungszustand schuld ist, worein die Muskulatur durch die Gefäßerweiterer versetzt wird, oder der gedehnte Zustand der Wandung an und für sich, muß vorläufig dahingestellt bleiben.

Nicht ohne Interesse erschien nun besonders die Frage, ob die Wirkungen des Stromes auf den Ort der Pole beschränkt bleiben oder sich fortpflanzen und, längere Strecken des Gefäßes ergreifen. Die beim Arbeiten am unverletzten Gewebe nicht zu vermeidenden Stromschleifen haben diese Prüfung vereitelt, doch verdient das gefundene Verhalten eine nähere Beschreibung, da man hier bei flüchtigem Beobachten leicht auf irrtümliche Deutungen geführt wird. Einige Einzelheiten der Versuchsanordnung werden hierbei mit anzuführen sein.

Folgende Einrichtung erwies sich als die beste. Die Elektrode, deren Wirkung beobachtet werden soll, wird von einer feinen öffnung in der durchsichtigen Deckplatte eines kleinen Beckens gebildet, welches mit physiologischer Kochsalzlösung gefüllt ist und durch ein seitlich einmündendes Röhrchen mit der Leitung in Verbindung steht ${ }^{1}$ ), indem dieses Rohr am anderen Ende in ein Gefäß mit Kochsalzlösung taucht; ein U-Röhrchen führt aus dieser in die Kupfer- oder Zinksulfatlösung, wodurch die Verbindung mit der Stromquelle hergestellt wird. Die Seitenwände des Kochsalzbeckens können aus einem paraffinierten Korkrähmchen bestehen, welches der Glasplatte des Experimentiertischchens aufgeklebt wird, die vom darunter aufgestellten Mikroskopspiegel das Licht empfängt; das Deckplättchen schneidet man am bequemsten aus Celluloid, das leicht zu durchbohren ist. Die Fugen zwischen Deckplättchen und Rahmen werden mit Paraffin sorgfältig abgedichtet. Auf dem Plättchen liegt die nicht zu feuchte Schwimmhaut des mit Urethan betäubten Frosches auf, ohne besondere Befestigung, so daß jedes Blutgefäß bequem an den Ort der Pore gebracht werden kann.

Am einfachsten jedoch nicht am zweckmäßigsten ist es nun, die andere Elektrode als indifferente einzurichten, indem der Frosch z. B. auf eine Metallplatte gelegt wird, die mit dem anderen Pol der Batterie verbunden ist ${ }^{2}$ ).

Bringt man nun die Pore unter den Stamm einer Arterie zu liegen, nicht zu weit von ihrem Ursprung aus dem Hauptgefäß der Zehe, so findet man einen aufälligen Unterschied in der Ausbreitung der kathodischen und der anodischen Wirkung. Während die kathodische Verengung auf das Stück der Arterie zunächst der Elektrode beschränkt

1) Die Pore als unpolarisierbare Elektrode ist von Pratt erfunden worden (The excitation of microscopic areas: a non-polarizable capillary electrode, Amer. Journ. of Physiol. 43, 159. 1917). Ich habe mich dieses Prinzipes bedient - ohne vorläufig mikroskopische Feinheit der Öffnung anzustreben, - und das Verfahren der Beobachtung im durchfallenden Lichte angepaßt.

2) Über den erforderlichen Strom möchte ich nur orientierend bemerken, daß die Effekte sehr empfindlich sind: bei dem bedeutenden Widerstand in der Zuleitung und im Tierkörper gab doch eine Spannung von einem Zehntelvolt bereits die ersten Spuren der Wirkung; die Pore hatte bei diesem Versuch einen Durchmesser von ungefähr $0,2 \mathrm{~mm}$. In der Regel verwendete ich Spannungen bis zu $10 \mathrm{Volt}$, doch hätten genauere Angaben keinen Wert, da ich keinen Versuch gemacht habe, die resultierende Stromdichte zu bestimmen. - Bei messenden Versuchen würde man natürlich auch die indifferente Elektrode unpolarisierbar anordnen. 
bleibt, breitet sich die anodische Erweiterung abwärts bis in die präcapillären Verzweigungen aus. Dies könnte entweder passive Entfaltung der distalen Teile oder einen wesentlichen Unterschied im Verhalten der beiden Pole vermuten lassen, doch trifft weder das eine noch das andere zu. Denn es müssen sich bei dieser unipolaren Einrichtung stets virtuelle Pole bilden, deren Lage man nicht beherrscht. Aus weiter nicht bekannten Gründen scheinen nun diese je nach der Stromrichtung etwas verschiedene Anordnung zu haben; jedenfalls machen sie sich häufig auf ungleiche Weise geltend. Ist die Elektrode anodisch, so entsteht proximalwärts sehr oft eine physiologische Kathode, unter: deren Einfluß sich die Arterie alsbald vollkommen verschließen kann, was der fortgesetzten Beobachtung der Anodenwirkung hinderlich ist. Hierin liegt der Nachteil der unipolaren Anordnung. Man sieht diese störende Kathode besonders dann deutlich, wenn die Pore nicht ganz oben am Ursprung der Arterie gelegen ist. - Distal von der Anode verhalten sich nun die Stromschleifen in der Regel so zu der Arterie, daß sie beim Eintritt die größere Dichte haben; die distalen Partien der Arterie werden daher erweitert. Ist die Elektrode dagegen kathodisch, so findet man die nun entstehenden virtuellen Anoden symmetrisch zur Pore gelegen, beide anliegenden Gebiete werden daher erweitert: man hat das Bild einer örtlichen Kathodenwirkung.

Worauf dieser Unterschied beruht, läßt sich, wie gesagt, nicht leicht angeben. Tatsächlich kommt es vor, $\mathrm{da} ß$ auch die anodische Wirkung lokal, in Gestalt einer spindelförmigen Erweiterung erscheint, der sanduhrförmigen Einschnürung entsprechend, die bei lokaler Kathodenwirkung $z \mathbf{u}$ sehen ist ${ }^{\mathfrak{l}}$ ).

Um die Stromschleifen nach Wunsch zu lenken, empfiehlt es sich nach dem Ausgeführten, auch die andere Elektrode an der Schwimmhaut selbst anzubringen, etwa in Gestalt einer Fadenelektrode. Bringt man nun die Pore weit unten an der Artexie an, die Fadenelektrode aber noch weiter distalwärts, an den Rand der Schwimmhaut, so sieht man, wie sich beide Wirkungen, die kathodische wie die anodische, weit hinauf bis ins Stammgebiet der Arterie ausbreiten. Auch wird nicht immer das Gefäß, das über der Pore liegt, allein betroffen; oft sieht man fast in der ganzen Schwimmhaut die entsprechenden gleichsinnigen Veränderungen an den Arterien. Indem so durch diese Beobachtung der scheinbare Unterschied in der Ausbreitung der kathodischen und der anodischen Wirkung wegfällt, läßt sich auf diesem Wege nichts beibringen, was auf eine Fortpflanzung der einen oder anderen von ihnen hindeutete, und wir werden sie bis auf weiteres als rein örtliche Reaktionen ansehen dürfen.

1) Man sieht diese Einschnürung besonders schön, wenn man eine sehr weite Arterie wählt, die von der Kathode nicht allzu stark verengt wird, so daß der Kreislauf ungestört bleibt. 